\title{
DFT-based low-complexity optimal cell ID estimation in NB-loT
}

Vincent Savaux

\begin{abstract}
This paper deals with cell identifier (ID) estimation for narrowband-Internet of things (NB-loT) system. It is suggested to transform the usual maximum likelihood (ML) estimator expression in order to highlight a discrete Fourier transform (DFT), which can be computed with fast algorithms. Therefore, the proposed method is a DFT-based low-complexity cell ID estimator that can be qualified as optimal in the ML sense. The principle is extended to the practical case where the channel is unknown and must be estimated. In this scenario, the concentrated likelihood function needs to be maximized, in which the ML channel estimate is a function of the unknown cell ID parameter. This operation only involves a few additional multiplications. Simulation results reveal that the performance of the proposed method actually matches the optimal one of the ML cell ID estimator. Furthermore, the technique is robust to residual frequency offset up to several hundreds of Hertz. We also show that the mean square error of channel estimation reaches its Cramér-Rao bound (CRB).
\end{abstract}

Keywords: NB-IoT, Maximum likelihood, DFT

\section{Introduction}

The Internet of things (IoT) market is growing rapidly as the number of applications increases in various domains such as industry, smart home, smart cities, and agriculture. Among the low-power wide area (LPWA) technologies allowing for long-range applications [1-3], narrowband-IoT (NB-IoT) is a promising solution as it is inherited from long-term evolution (LTE) [4-7]. Thus, similar to LTE, NB-IoT devices work in licensed frequency bands, occupying one resource block of the LTE system. Moreover, every device must be synchronized with an evolved node B (eNB) to connect the network. The synchronization process is carried out according to two main steps: the devices physically synchronize with the eNBs using the narrowband primary synchronization signal (NPSS) [8-11] and then seek the cell identifier (ID) of the neighboring eNBs through the narrowband secondary synchronization signal (NSSS). In this paper, we focus on the cell ID estimation process.

Correspondence: vincent.savaux@b-com.com

IRT $b<>$ com, Rennes, France
The cell ID can take 504 different values and is transmitted through the NSSS as a combination (multiplication) of a Zadoff-Chu (ZC) sequence and a Hadamard sequence. Few papers dealing with the cell ID estimation in NB-IoT have been proposed in the literature yet. In [12], the exhaustive maximum likelihood (ML) cell ID search is suggested, and computational simplifications are established in $[13,14]$. The ML leads to a cross-correlations between the observations (i.e., the received NSSS) and all the possible combinations of ZC and Hadamard sequences. The presented reductions of complexity in $[13,14]$ are based on the fact that the Hadamard sequences composing the NSSS consist in \pm 1 elements. As a consequence, all the multiplications of the NSSS observation by the Hadamard sequences do not need to be computed as it only changes the sign of the observation samples. Despite their advantageous reduction of complexity, the methods in $[13,14]$ are based on cross-correlation and do not take further advantage of the NSSS features to simplify the cell ID estimation.

In this paper, we suggest to rewrite the cross-correlation in the cell ID ML estimator as a discrete Fourier transform (DFT) of the observations. To do so, we first notice 
that the ZC sequence consists in complex exponential samples that can then be transformed and reformulated as the coefficients $\omega^{\mathrm{kn}}=e^{-2 j \pi \frac{\mathrm{kn}}{N}}$ of the DFT. It results that algorithms for fast computations of DFTs $[15,16]$ can be used, therefore reducing the complexity of the ML estimator. Furthermore, the suggested method is also compatible with other simplifications based on the property of Hadamard sequences [13, 14]. In addition, we deal with the practical case where the channel is unknown and must be jointly estimated with the cell ID. It is proved that the complex argument of the channel only needs to be estimated, as the modulus of the channel is not involved in the maximization of the likelihood function. Besides, we show that the cell ID can then be estimated by maximizing the so-called concentrated-likelihood function in which the channel argument is substituted by the estimated one and whose value only depends on the cell ID value. This operation only slightly increases the complexity of the proposed ML estimator. The simulation results show that the performance of the suggested method actually matches that of the exhaustive ML and that it remains accurate for residual frequency offset of several hundreds of Hertz. Moreover, it is verified through simulations that the mean square error (MSE) of the estimator of the channel phase reaches the Cramér-Rao bound (CRB).

The rest of the paper is organized as follows: Section 3 presents the NSSS reception model, including the exhaustive ML cell ID search. Section 4 introduces the suggested low-complexity ML estimator, and it is extended to the practical case where the channel needs to be estimated. The performance of the estimator is shown and discussed in Section 5, and Section 6 concludes this paper.

Notation: the vectors and matrices are written in boldface $\mathbf{x}$ and uppercase boldface $\mathbf{X}$, respectively, and the scalars are written in normal font $x$. $\|$.$\| represents the$ Euclidian norm, |.| the modulus (of complex scalar), and $\operatorname{Re}\{$.$\} the real part. Moreover, x^{*}, \arg ($.$) , and (.. { }^{H}$ stand for the conjugate, the argument, and the Hermitian (complex conjugate) operators, respectively. The modulo is written mod, the mathematical expectation is denoted by $\mathbb{E}\{$.$\} ,$ and $\otimes$ is the Kronecker product.

\section{Method}

The aim of this study is to prove that the ML estimation of the cell ID in NB-IoT system can be carried out through a simple DFT without loss of performance compared with the exhaustive search. The paper includes a theoretical development that describes the DFT-based ML cell ID estimator and simulation results that show the relevance of the suggested method. The theoretical study does not require any specific material, and the simulations have been carried out with MatLab R2016a.

\section{NSSS reception}

\subsection{System model}

This section describes the received NSSS, which is also detailed in [17]. The NSSS is composed of 11 OFDM symbols of 12 subcarriers, namely $11 \times 12=132$ resource elements. It is assumed that the time-frequency synchronization has been accurately performed thanks to the NPSS [8-11], in such way that the residual synchronization errors are negligible. Otherwise, the effect of the potential residual errors will be discussed in Section 5. Furthermore, since the signal is narrowband, it is reasonable to suppose that the frequency response of the channel is constant over the NSSS resource block of 12 subcarriers. Thus, after the cyclic prefix removal and the DFT over the 11 OFDM symbols, the received NSSS column vector y of size $N=132$ can be expressed as

$$
\mathbf{y}=\alpha \mathbf{d}+\mathbf{w},
$$

where $\alpha$ is the complex channel coefficient, $\mathbf{w}$ is the additive white Gaussian noise (AWGN) vector of size $N \times$ 1 containing the samples $w_{n} \sim \mathcal{C N}\left(0, \sigma^{2}\right)$, and $\mathbf{d}$ is the transmitted NSSS sequence, whose elements $d_{n}$ are defined in [17] as

$$
d_{n}=b_{q}(m) e^{-2 j \pi \theta_{f} n} e^{-j \frac{\pi u n^{\prime}\left(n^{\prime}+1\right)}{131}},
$$

where $\left\{b_{q}(m) \in\{-1,1\}\right\}$ is one of the four Hadamard sequences defined in Table 10.2.7.2.1-1 in [17], which has been reproduced in Table 1. Moreover, we have

$$
\theta_{f}=\frac{1}{4}\left(\left(\frac{n_{f}}{2}\right) \bmod 4\right)
$$

Table 1 Definition of $b_{q}(m)$

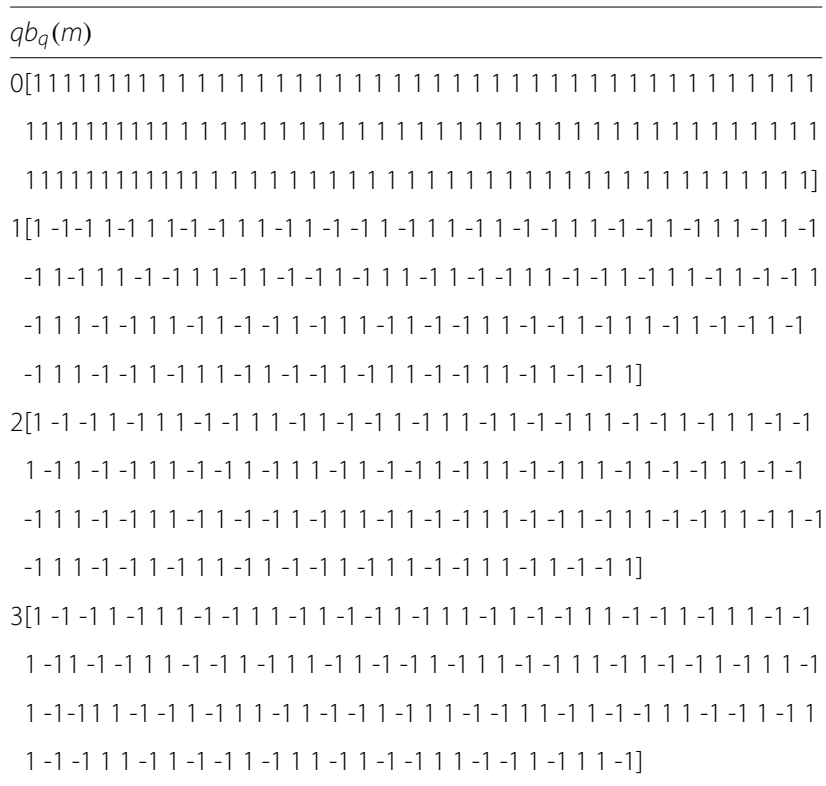


where $n_{f}$ is the frame number, which is always even. Furthermore, we have:

$$
\begin{aligned}
& n^{\prime}=n \bmod 131 \\
& m=n \bmod 128 \\
& u=N_{\text {ID }}^{\text {Ncell }} \bmod 126+3 \\
& q=\left\lfloor\frac{N_{\text {ID }}^{\text {Ncell }}}{126}\right\rfloor,
\end{aligned}
$$

with $N_{\mathrm{ID}}^{\mathrm{Ncell}}$ the cell ID of the eNB.

For a sake of clarity, we can rewrite (1) in a detailed form as follows:

$$
\mathbf{y}=\alpha \underbrace{\mathbf{B} \boldsymbol{\theta}}_{\mathbf{X}\left(q, \theta_{f}\right)} \mathbf{e}(u)+\mathbf{w},
$$

where $\mathbf{B}, \boldsymbol{\theta}$, and $\mathbf{X}\left(q, \theta_{f}\right)$ are the $N \times N$ diagonal matrix composed of the elements $b_{q}(m), e^{-2 j \pi \theta_{f} n}$, and $b_{q}(m) e^{-2 j \pi \theta_{f} n}$, respectively. Note that the diagonal elements of $\mathbf{X}\left(q, \theta_{f}\right)$ are taken from a finite set $\{ \pm 1, \pm j\}$. The $N \times 1$ vector $\mathbf{e}(u)$ contains the ZC sequence $[18,19]$ $e_{n}(u)=e^{-j \frac{\pi u n^{\prime}\left(n^{\prime}+1\right)}{131}}$, where the parameter $u$ is called the root of the sequence. In the following, the estimation of the cell ID results in finding the parameters $(u, q)$ from the observation $\mathbf{y}$, as the cell ID can be expressed from (4) as

$$
N_{\text {ID }}^{\text {Ncell }}=126 q+u-3 .
$$

It can be noticed that the estimation of the cell ID does not require that of the parameter $\theta$, but it must be nevertheless properly estimated in order to unscramble the received NSSS sequence, i.e., remove the sequence $\boldsymbol{\theta}$.

\subsection{Maximum likelihood cell ID estimation}

The ML estimation of $u, q$, and $\theta_{f}$ leads to an exhaustive search of the optimal parameters through the likelihood function of complex observation $\mathbf{y}$, denoted by $L$, which can be expressed as

$$
\left(\hat{u}, \hat{q}, \hat{\theta}_{f}\right)=\underset{u, q, \theta_{f}}{\arg \max } \underbrace{C e^{-\frac{1}{\sigma^{2}}\left\|\mathbf{y}-\alpha \mathbf{X}\left(q, \theta_{f}\right) \mathbf{e}(u)\right\|^{2}}}_{L},
$$

where $C$ is a constant that can be omitted in the following developments since it does not depend on $\left(u, q, \theta_{f}\right)$. Since $\left|X_{n}\right|^{2}=1$ and $\left|e_{n}(u)\right|^{2}=1$ for any $X_{n} \in\{ \pm 1, \pm j\}$, we can develop (7) as

$$
\begin{aligned}
\left(\hat{u}, \hat{q}, \hat{\theta}_{f}\right)= & \underset{u, q, \theta_{f}}{\arg \max } e^{-\frac{1}{\sigma^{2}}\|\mathbf{y}\|^{2}} e^{-\frac{|\alpha|^{2} N}{\sigma^{2}}} \\
& \times e^{\frac{2}{\sigma^{2}} \operatorname{Re}\left\{\mathbf{y}^{H} \alpha \mathbf{X}\left(q, \theta_{f}\right) \mathbf{e}(u)\right\}} \\
= & \underset{u, q, \theta_{f}}{\arg \max } \operatorname{Re}\left\{\mathbf{y}^{H} \alpha \mathbf{X}\left(q, \theta_{f}\right) \mathbf{e}(u)\right\},
\end{aligned}
$$

which can be rewritten in a scalar form as

$$
\left(\hat{u}, \hat{q}, \hat{\theta}_{f}\right)=\underset{u, q, \theta_{f}}{\arg \max } \operatorname{Re}\left\{\sum_{n=0}^{131} y_{n}^{*} \alpha X_{n}\left(q, \theta_{f}\right) e_{n}(u)\right\} .
$$

The cross-correlation in (9) requires $N_{m}=16 \times 126 \times$ $132=266112$ complex multiplications, where 16 corresponds to $q \times \theta_{f}$ possible values, and 126 corresponds to $u$ possible values. Furthermore, it requires the a priori knowledge of the channel coefficient $\alpha$ or it must be estimated. In the following, we take advantage of the ZC sequence $e_{n}(u)=e^{-j \frac{\pi u n^{\prime}\left(n^{\prime}+1\right)}{131}}$ to rewrite (9) as a DFT, therefore simplifying the process. Furthermore, the estimation of the channel is tackled as well.

\section{Suggested low-complex ML cell ID estimation}

This section presents the way to reformulate the ML estimator (9) into a simplest form using DFT. The principle of the method is first detailed in an ideal case where the channel is supposed to be known (or at least accurately estimated), and we then extend to a more general case where both the cell ID and the channel are estimated through ML using DFT formulation.

\subsection{Ideal case: known channel}

In order to reduce the complexity of the cell ID estimation, we can rewrite (9) by means of a DFT. For clarity purpose, we note $z_{n}=y_{n}^{*} \alpha X_{n}\left(q, \theta_{f}\right)$, then (9) can be expressed as

$$
\begin{aligned}
\left(\hat{u}, \hat{q}, \hat{\theta}_{f}\right) & =\underset{u, q, \theta_{f}}{\arg \max } \operatorname{Re}\left\{\sum_{n=0}^{131} z_{n} e^{-\frac{j \pi u n^{\prime}\left(n^{\prime}+1\right)}{131}}\right\} \\
& =\underset{u, q, \theta_{f}}{\arg \max } \operatorname{Re}\left\{\sum_{n=0}^{131}\left|z_{n}\right| e^{j \arg \left(z_{n}\right)} e^{-\frac{j \pi u n^{\prime}\left(n^{\prime}+1\right)}{131}}\right\} .
\end{aligned}
$$

To further develop the sum in (10), it should be noticed that $n^{\prime}\left(n^{\prime}+1\right)$ is always even, for every even or odd $n^{\prime}$ and can be expressed as

$$
n^{\prime}\left(n^{\prime}+1\right)=2\left(\begin{array}{c}
n^{\prime}+1 \\
2
\end{array}\right)
$$

To recognize a DFT, we define $\tilde{n}=\left(\begin{array}{c}n^{\prime}+1 \\ 2\end{array}\right) \bmod 131$ such that the sum in (10) can be rewritten as

$$
\sum_{n=0}^{131}\left|z_{n}\right| e^{j \arg \left(z_{n}\right)} e^{-\frac{j \pi u n^{\prime}\left(n^{\prime}+1\right)}{131}}=\sum_{n=0}^{131}\left|z_{n}\right| e^{j \arg \left(z_{n}\right)} e^{-\frac{2 j \pi u \tilde{n}}{131}}
$$

We show in Fig. $1 \tilde{n}=n^{\prime}\left(n^{\prime}+1\right) \bmod 131$ versus $n$, with $n^{\prime}=n \bmod 131$. Thus, we can observe that $\tilde{n}$ is symmetrical according to $n=65$, i.e., for any $n_{1}<65$, there exists a $65<n_{2} \leq 130$ such as $n_{1}+n_{2}=130$ and $\tilde{n}_{1}=\tilde{n}_{2}$. Moreover, the set $\Omega_{n}=\left\{\tilde{n}=\left(\begin{array}{c}n^{\prime}+1 \\ 2\end{array}\right)\right.$ $\bmod 131 \mid n=0,1, . ., N-1\}$ is a subset of $[[0,131]]$. As a consequence, we can rearrange the elements $\left|z_{n}\right| e^{j \arg \left(z_{n}\right)}$ 


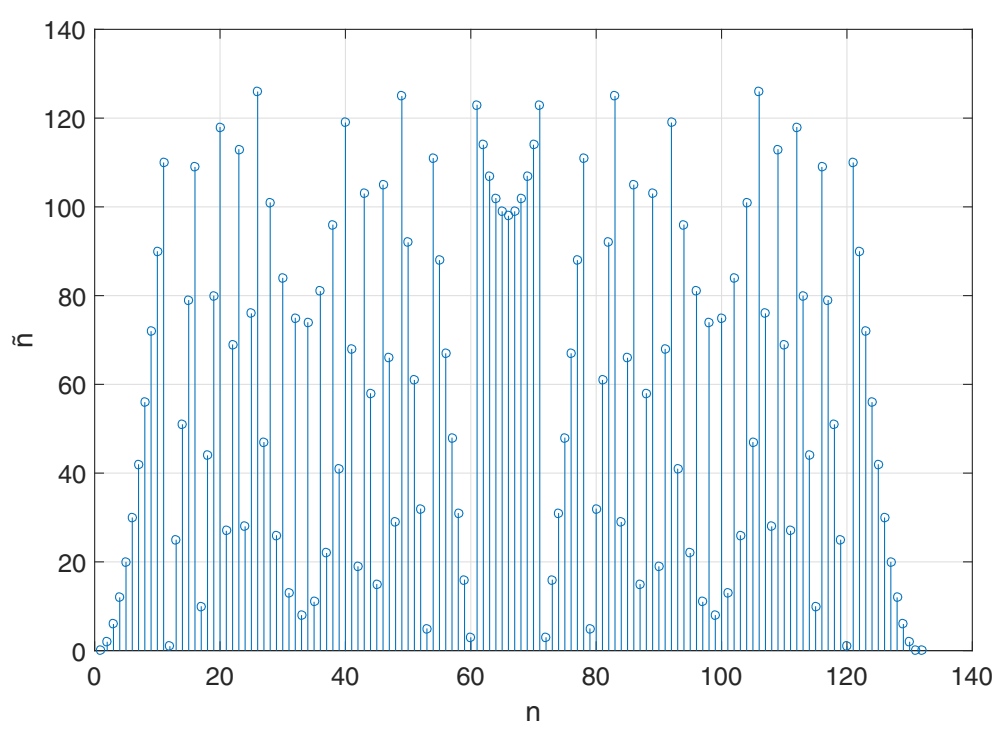

Fig. $1 \tilde{n}=n^{\prime}\left(n^{\prime}+1\right) \bmod 131$ versus $n$, with $n^{\prime}=n \bmod 131$

in order to highlight the DFT. To this end, we define $p_{k}$ as

$$
p_{k}=\left\{\begin{array}{l}
\left|z_{0}\right| e^{j \arg \left(z_{0}\right)}+\left|z_{130}\right| e^{j \arg \left(z_{130}\right)} \\
+\left|z_{131}\right| e^{j \arg \left(z_{131}\right)}, \text { if } k=\tilde{n}=0 \\
\left|z_{n_{1}}\right| e^{j \arg \left(z_{n_{1}}\right)}+\left|z_{n_{2}}\right| e^{j \arg \left(z_{n_{2}}\right)}, \\
\text { if } k=\tilde{n}_{1} \in\left\{\Omega_{n} \backslash\{0\}\right\}, n_{1}+n_{2}=130 \\
0, \text { if } \mathrm{k} \notin \Omega_{n}
\end{array} .\right.
$$

Finally, from (12) and the rearrangement in (13), we obtain:

$$
\sum_{n=0}^{131}\left|y_{n}\right| e^{j \arg \left(z_{n}\right)} e^{-\frac{2 j \pi u \tilde{n}}{131}}=\sum_{k=0}^{130} p_{k} e^{-\frac{2 j \pi u k}{131}},
$$

where we recognize the DFT of $p_{k}$. By defining the vector $\mathbf{p}=\left[p_{0}, p_{1}, \ldots, p_{N-1}\right]$, the ML estimator of $u, q$, and $\theta_{f}$ can be simply expressed as

$$
\left(\hat{u}, \hat{q}, \hat{\theta}_{f}\right)=\underset{u, q, \theta_{f}}{\arg \max } \operatorname{Re}\{D F T(\mathbf{p})\} .
$$

Figure 2 illustrates $\operatorname{Re}\{D F T(\mathbf{p})\}$ versus $u$, for a ZC sequence featuring a root $u=7$. We can see a peak at

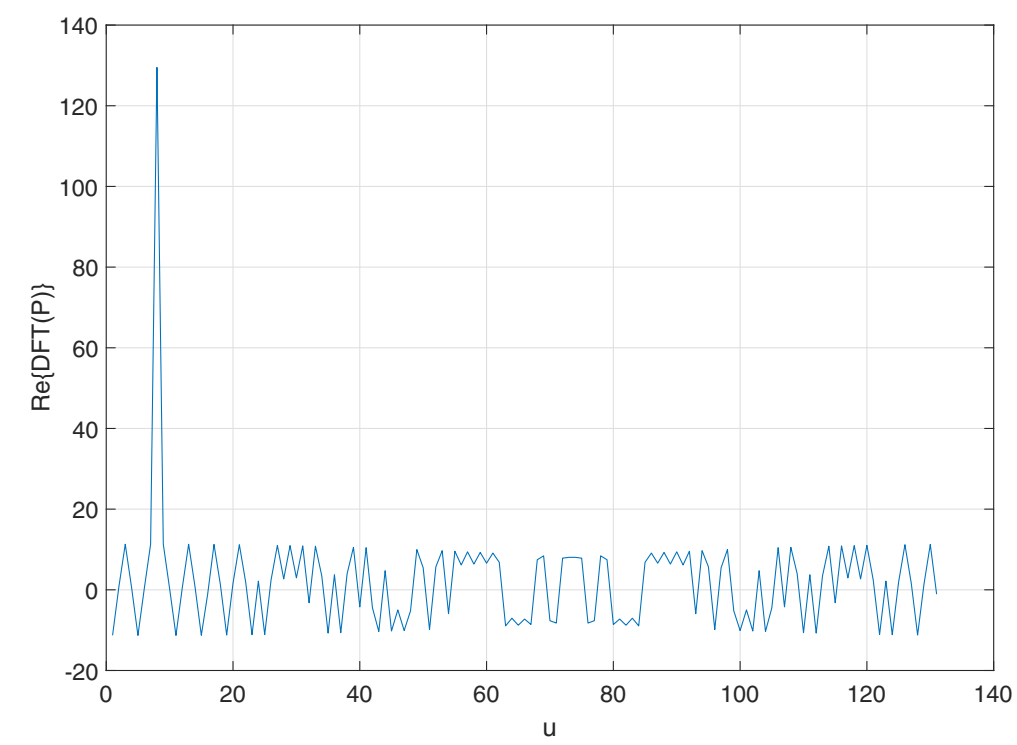

Fig. $2 \operatorname{Re}\{D F T(\mathbf{p})\}$ versus $u, Z C$ sequence has root $u=7$ 
$u=7$, which proves the relevance of the suggested transformation. The direct computation of (15) using DFT has a complexity of $16 \times 66 \times 126=133056$ complex multiplications, which is very similar to the exhaustive search in (9). This is because 131 is a prime number. However, this can be reduced by using an algorithm for fast calculation of prime DFT, such as FFTW ${ }^{1}$, chirp-z algorithm [15], or generalized Goertzel algorithm [16]. Thus, considering the complexity analysis of the chirp- $\mathrm{z}$ algorithm [15] for instance, the computation of (15) requires 2 multiplications of size 132, one of size 256 (the first power of 2 larger than 132), and 2 FFTs of size 256. Therefore, the complexity of (15) is of order $16 \times 4016=73856$ where 16 correspond to the estimation of $\left(u, \theta_{f}\right)$ and 4016 to the chirp-z algorithm. This is of order four times less complex than the exhaustive search (9) and could be even simplified. In fact, it must be noted that the FFT is sparse, as only 66 samples over 131 are non-zero in the input vector $\mathbf{p}$; therefore, numerous multiplications can be avoided. Furthermore, we can take advantage of the fact that $X_{n}\left(q, \theta_{f}\right) \in\{ \pm 1, \pm j\}$ to further simplify (15) since the 16 possible combinations $\left(q, \theta_{f}\right)$ can be tested with straightforward changes of signs in the different sums that compose the FFTs. The complexity could then be reduced by eight, namely 9232 multiplications, leading to a computation cost similar to that in $[13,14]$. However, note that the different FFT implementations are out of the scope of this paper and then not further dealt with in this work. In the following, we extend the suggested solution to a more realistic case where the channel is unknown and must be estimated.

\subsection{ML cell ID and channel estimation}

From (9), we can notice that the substitution $\alpha=|\alpha| e^{j \phi_{\alpha}}$ with $\phi_{\alpha}$ the argument of $\alpha$, yields:

$$
\left(\hat{u}, \hat{q}, \hat{\theta}_{f}\right)=\underset{u, q, \theta_{f}}{\arg \max R e}\left\{\sum_{n=0}^{131} y_{n}^{*} e^{j \phi_{\alpha}} X_{n}\left(q, \theta_{f}\right) e_{n}(u)\right\},
$$

since $|\alpha|$ is independent of $\left(u, q, \theta_{f}\right)$ and can then be removed from (9). We deduce that it is sufficient to focus on the estimation of $\phi_{\alpha}$ to, in turn, estimate $\left(\hat{u}, \hat{q}, \hat{\theta}_{f}\right)$. Thus, the ML estimation of $\phi_{\alpha}$ can be expressed, after straightforward developments, as

$$
\begin{aligned}
\hat{\phi}_{\alpha} & =\max _{\phi_{\alpha}} L \\
& =\max _{\phi_{\alpha}} \underbrace{\operatorname{Re}\left\{\sum_{n=0}^{131} y_{n}^{*} e^{j \phi_{\alpha}} X_{n}\left(q, \theta_{f}\right) e_{n}(u)\right\}}_{f\left(\phi_{\alpha}\right)} .
\end{aligned}
$$

Hence, solving $\frac{\partial}{\partial \phi_{\alpha}} f\left(\phi_{\alpha}\right)=0$ leads to

$$
\begin{gathered}
e^{2 j \phi_{\alpha}}=\frac{\sum_{n=0}^{131} y_{n} X_{n}^{*}\left(q, \theta_{f}\right) e_{n}^{*}(u)}{\sum_{n=0}^{131} y_{n}^{*} X_{n}\left(q, \theta_{f}\right) e_{n}(u)} \\
\Rightarrow \hat{\phi}_{\alpha}=\frac{1}{2} \arg \left(\frac{\sum_{n=0}^{131} y_{n} X_{n}^{*}\left(q, \theta_{f}\right) e_{n}^{*}(u)}{\sum_{n=0}^{131} y_{n}^{*} X_{n}\left(q, \theta_{f}\right) e_{n}(u)}\right) \\
\Leftrightarrow \hat{\phi}_{\alpha}=\arg \left(\sum_{n=0}^{131} y_{n} X_{n}^{*}\left(q, \theta_{f}\right) e_{n}^{*}(u)\right),
\end{gathered}
$$

since the denominator is the conjugate of the numerator. The CRB $[20,21]$ of this estimator is given by $C R B=\frac{\sigma^{2}}{2|\alpha|^{2} N}$, such as proved in Appendix 6. In the following, we note the estimate $\hat{\phi}_{\alpha}\left(u, q, \theta_{f}\right)$ in order to highlight its dependency to the unknown discrete parameters. Furthermore, we can rewrite the ML estimator (16) by substituting the likelihood function $L$ by the concentrated-likelihood one (including the estimate $\left.\hat{\phi}_{\alpha}\left(u, q, \theta_{f}\right)\right)$, leading to

$$
\left(\hat{u}, \hat{q}, \hat{\theta}_{f}\right)=\underset{u, q, \theta_{f}}{\arg \max R e}\left\{e^{j \hat{\phi}_{\alpha}\left(u, q, \theta_{f}\right)} \sum_{n=0}^{131} y_{n}^{*} X_{n}\left(q, \theta_{f}\right) e_{n}(u)\right\} .
$$

Interestingly, it must be noticed that the sum $\sum_{n=0}^{131} y_{n}^{*} X_{n}\left(q, \theta_{f}\right) e_{n}(u)$ is found in both (18) and (19). It can then be computed only once by using the DFT-based method leading to (15) with $z_{n}=y_{n}^{*} X_{n}\left(q, \theta_{f}\right)$ and (19) then becomes

$$
\left(\hat{u}, \hat{q}, \hat{\theta}_{f}\right)=\underset{u, q, \theta_{f}}{\arg \max } \operatorname{Re}\left\{e^{j \hat{\phi}_{\alpha}\left(u, q, \theta_{f}\right)} D F T(\mathbf{p})\right\} .
$$

The joint ML cell ID and channel estimation algorithm can be summarized as follows:

1. Compute $D F T(\mathbf{p})$ with $z_{n}=y_{n}^{*} X_{n}\left(q, \theta_{f}\right)$ by using an algorithm such as the chirp-z transform. This step has the same complexity as that discussed after (15).

2. Estimate $\hat{\phi}_{\alpha}\left(u, q, \theta_{f}\right)$ in (18). The computation of $\hat{\phi}_{\alpha}\left(u, q, \theta_{f}\right)$ requires the ratio of the real and the imaginary parts of $\sum_{n=0}^{131} y_{n} X_{n}^{*}\left(q, \theta_{f}\right) e_{n}^{*}(u)$ to feed the arc-tangent function. The computational cost is then $16 \times 126=2016$ multiplications, corresponding to all possible $\left(u, q, \theta_{f}\right)$ values.

3. Estimate $\left(u, q, \theta_{f}\right)$ in (20). This also requires 2016 multiplications.

The general algorithm for joint cell ID and channel estimation then requires 4032 more complex multiplications than the ideal case where the channel is supposed to be known. This is a reasonable additional complexity compared to the ML, even in simplified implementations such as the suggested one or those presented in [13, 14]. Table 2 summarizes the achievable complexity of the 
Table 2 Complexity comparison of the suggested DFT-based cell ID estimator with [12-14], in a number of complex multiplications

\begin{tabular}{lll} 
& Cell ID & Channel \\
\hline Exhaustive ML [12] & 266112 & N/A \\
{$[13]$} & 66528 & N/A \\
{$[14]$} & 16632 & N/A \\
Suggested ML & $<73856^{(1)}$ & 4032 \\
\hline
\end{tabular}

Notes: (1) This value is a loose upper bound of the achievable complexity. Such as aforementioned, this could be reduced by 8, namely 9232

suggested DFT-based cell ID estimator compared with [12-14], given in a number of complex multiplications. The indicated value 73856 is given considering the chirp$\mathrm{z}$ algorithm for the computation of the DFT. In that case, the computation cost is of the same order as [13]. Moreover, if we consider further simplifications (sparse DFT and $X_{n}\left(q, \theta_{f}\right) \in\{ \pm 1, \pm j\}$, not dealt with in this paper), the complexity could be reduced to 9232 , i.e., of the same order as [14]. In addition, the channel estimation has been added as well, which is not considered in [12-14].

Unfortunately, we cannot theoretically predict the estimation performance in case of discrete unknown parameters (except in binary detection), such as done through CRB for continuous parameters. In fact, it is only tractable to asymptotically predict the probability of estimation errors, such as hereby presented. As a consequence, we will show the accuracy of the estimators (15) and (20) through simulations in Section 5.

\subsection{Asymptotic error probability analysis}

In this section, we carry out an asymptotic analysis of the probability of error of cell ID estimation for the ML estimators (9) and its DFT-based version (15). An error of cell ID estimation occurs if $q, u$, or both parameters are badly estimated. We denote by $\mathcal{P}$ this probability, then it is expressed as

$$
\begin{aligned}
\mathcal{P}= & \mathbb{P}\left(\hat{N}_{\mathrm{ID}}^{\mathrm{Ncell}} \neq N_{\mathrm{ID}}^{\mathrm{Ncell}}\right) \\
= & \mathbb{P}(\hat{u}=u \cap \hat{q} \neq q)+\mathbb{P}(\hat{u} \neq u \cap \hat{q}=q) \\
& +\mathbb{P}(\hat{u} \neq u \cap \hat{q} \neq q) .
\end{aligned}
$$

We can reasonably assume that the events are independent (this assumption will be verified in Section 5), therefore $\mathcal{P}$ simplifies to

$$
\begin{aligned}
\mathcal{P}= & \mathbb{P}(\hat{u}=u) \mathbb{P}(\hat{q} \neq q)+\mathbb{P}(\hat{u} \neq u) \mathbb{P}(\hat{q}=q) \\
& +\mathbb{P}(\hat{u} \neq u) \mathbb{P}(\hat{q} \neq q) \\
= & \mathbb{P}(\hat{q} \neq q)+\mathbb{P}(\hat{u} \neq u) \mathbb{P}(\hat{q}=q) .
\end{aligned}
$$

Unfortunately, the probabilities $\mathbb{P}(\hat{q} \neq q)$ and $\mathbb{P}(\hat{u} \neq$ $u$ ) (respectively $\mathbb{P}(\hat{q}=q)$ and $\mathbb{P}(\hat{u}=u)$ ) do not have tractable expressions, as they involve multiple integrals of multivariate Gaussian distributions. However, we can straightforwardly obtain the upper bound of the probabilities in (22) when $\sigma^{2}$ tends to $+\infty$. In that case, the events $\hat{q}=q$ and $\hat{u}=u$ are equiprobable for any $q$ and $u$, respectively. As a consequence, we have $\lim _{\sigma^{2} \rightarrow+\infty} \mathbb{P}(\hat{q} \neq q)=\frac{3}{4}$ and $\lim _{\sigma^{2} \rightarrow+\infty} \mathbb{P}(\hat{u} \neq u)=$ $\frac{125}{126}$, yielding $\lim _{\sigma^{2} \rightarrow+\infty} \mathcal{P}=\frac{503}{504}$. In fact, this corresponds to the probability of missing the cell ID in a random choice of the cell ID among the 504 possible values.

\section{Simulations and discussion}

\subsection{Simulations results}

The simulation results have obtained using MatLab, and $10^{5}$ independent runs per point have been performed. The channel coefficient obeys a zero-mean complex Gaussian distribution with unitary variance. The signal to noise ratio (SNR) is defined as SNR = $\frac{\mathbb{E}\left\{\|\alpha \mathbf{d}\|^{2}\right\}}{\mathbb{E}\left\{\|\mathbf{w}\|^{2}\right\}}$. In the simulations, the chirp-z algorithm [15] has been used, but we remind that other fast DFT techniques could be applied. Note that for simplicity matter, we use a descriptive shortcut in all the following comments. Thus, we mention the "ML cell ID estimation" whereas we refer to the ML estimations of $\left(u, q, \theta_{f}\right)$ (e.g., in (9) or (15)). The actual cell ID estimation is obtained from the estimate $(\hat{u}, \hat{q})$ using (6), i.e., $\hat{N}_{\mathrm{ID}}^{\mathrm{Ncell}}=126 \hat{q}+\hat{u}-3$.

\subsubsection{Known channel}

In Fig. 3, we compare the ML exhaustive cell ID search (9) and the suggested DFT-based implementation (15) through the probability of error of estimation versus SNR in the range $[-15,-2] \mathrm{dB}$. We assume the ideal case where the channel is known. It can be verified that both trajectories match, as a very slight difference $(<0.1 \mathrm{~dB})$ can be observed. The latter can be due to the computation in transformed domains that are involved in the suggested algorithm. In fact, it can be seen for instance in Fig. 2 that $\operatorname{Re}\{D F T(\mathbf{p})\}$ is not null around the peak at $u=7$, which may lead to few errors in low SNR range. However, this result shows that the suggested implementation of the ML estimator does not change its performance as the difference is negligible. We can observe that the probability of estimation error dives below $10^{-3}$ for SNR values larger than $-7.5 \mathrm{~dB}$, showing the robustness of the $\mathrm{ML}$ estimator.

Figure 4 shows $\mathcal{P}$ versus SNR from -20 to $-8 \mathrm{~dB}$, the different probabilities of error developed in (21) and (22) and the corresponding asymptotes in very low SNR range. It can be observed that it is more likely to badly estimate both $u$ and $q$ than one of the parameter for any SNR value. Moreover, the asymptotes $0.744,0.248$, and 0.006 well fit $\mathbb{P}(\hat{u} \neq u \cap \hat{q} \neq q), \mathbb{P}(\hat{u} \neq u \cap \hat{q}=q)$, and $\mathbb{P}(\hat{u}=u \cap \hat{q} \neq$ $q$ ), respectively, in a very low SNR range. This shows that the assumption of independence of the events holds, as for 


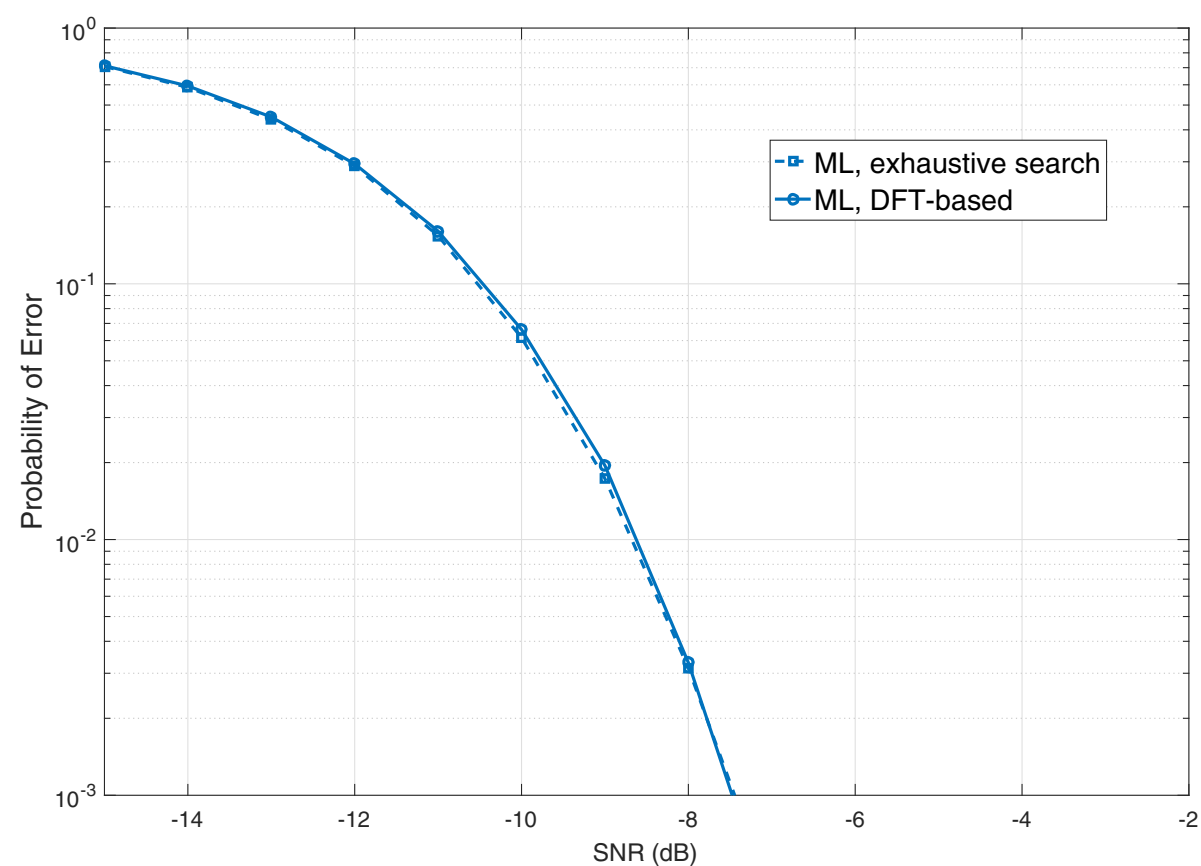

Fig. 3 Error probability versus SNR (dB) of cell ID ML estimator using (9) and the suggested DFT-based implementation (15). A known channel is assumed

instance

$$
\begin{aligned}
\lim _{\sigma^{2} \rightarrow+\infty} \mathbb{P}(\hat{u} \neq u \cap \hat{q} \neq q) & =\lim _{\sigma^{2} \rightarrow+\infty} \mathbb{P}(\hat{u} \neq u) \mathbb{P}(\hat{q} \neq q) \\
& =\frac{125}{126} \times \frac{3}{4}=0.744
\end{aligned}
$$

The assumption of independence of the events is also verified in Fig. 4 as we can see that (22) holds. In fact, the behaviors of $\mathcal{P}$ and $\mathbb{P}(\hat{q} \neq q)+$ $\mathbb{P}(\hat{u} \neq u) \mathbb{P}(\hat{q}=q)$ exactly match for any SNR value.

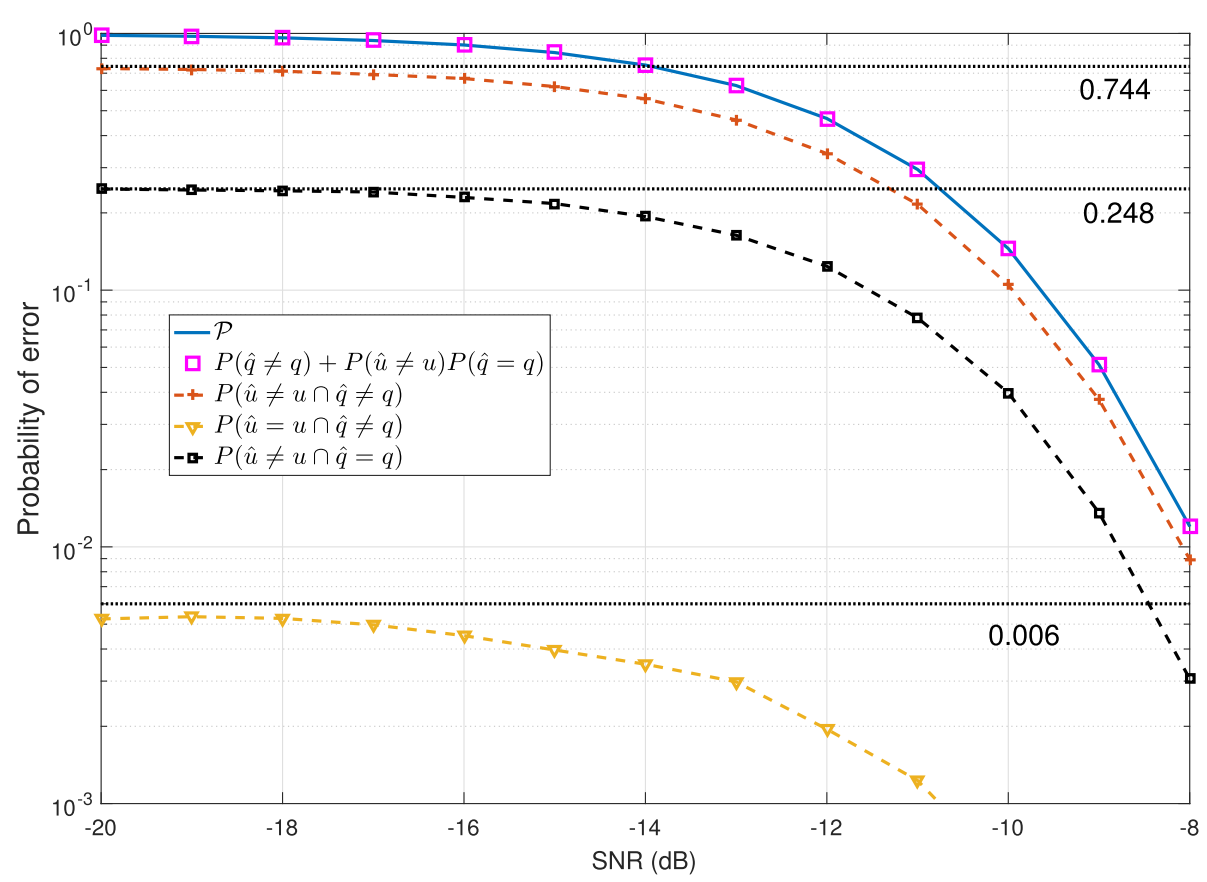

Fig. 4 Probabilities of error versus SNR (dB), $\mathcal{P}$ and developments from (21), (22), and asymptotes 


\subsubsection{Estimated channel}

Figure 5 compares the probability of error of the suggested cell ID estimation versus SNR in the ideal cases where the channel is supposed to be known with the more practical case where the channel phase is estimated. It can be observed that the performance of the cell ID estimation where the channel is estimated is only $0.8 \mathrm{~dB}$ weaker than the ideal case. This shows that the substitution of the likelihood function by the concentrated-likelihood function only slightly degrades the performance and that the channel phase is accurately estimated in (18).

In order to confirm the previous statement, we show in Fig. 6 the MSE of the channel phase estimator versus SNR, where the MSE is defined as

$$
\mathrm{MSE}=\mathbb{E}\left\{\left|\hat{\phi}_{\alpha}-\phi_{\alpha}\right|^{2}\right\} .
$$

Moreover, it is compared with the CRB previously defined. It can be seen in Fig. 6 that the MSE matches the $\mathrm{CRB}$, the phase estimator is then optimal in the ML sense.

\subsection{Discussion}

\subsubsection{Performance of the method under residual frequency offset hypothesis}

In this section, we consider the non-idealistic case where a residual frequency offset still remains after the physical synchronization stage thanks to NPSS. Thus, Fig. 7 shows the performance of the suggested ML cell ID estimator (20) (i.e., the channel phase is estimated) in the presence of a phase offset $\Delta_{f}$ taken in the set $\{100,200,400\} \mathrm{Hz}$. We can observe in Fig. 7 that the estimation error behaviors corresponding to $\Delta_{f}=100,200$, and $400 \mathrm{~Hz}$ experience performance losses of $0.1,0.4$, and $1.3 \mathrm{~dB}$, respectively. These results show the robustness of the DFT-based ML cell ID estimator to the residual errors, in particular, if it is below $200 \mathrm{~Hz}$.

\subsubsection{Sub-optimal ML-based estimator}

In order to avoid the channel phase estimation step, we suggest to rewrite (20) by substituting the real part by the modulus operator as follows:

$$
\begin{aligned}
\left(\hat{u}, \hat{q}, \hat{\theta}_{f}\right) & =\underset{u, q, \theta_{f}}{\arg \max }\left|e^{j \hat{\phi}_{\alpha}\left(u, q, \theta_{f}\right)} \operatorname{DFT}(\mathbf{p})\right| \\
& =\underset{u, q, \theta_{f}}{\arg \max }|\operatorname{DFT}(\mathbf{p})| .
\end{aligned}
$$

This new expression is referred as the "alternative form" of the ML estimator. It is supposed to be sub-optimal compared with the DFT-based ML method in (20), as it is not obtained from the maximization of the (concentrated) likelihood function. However, it is shown in Fig. 8 that the performance of the alternative form actually matches that of the DFT-based ML estimator. This could be due to the channel model we assumed: we considered an invariant channel over 12 subcarriers. Thus, taking the modulus removes the channel phase in the expression of the estimator. We draw from Fig. 8 that in very low frequency selectivity conditions, if the channel does not need to be

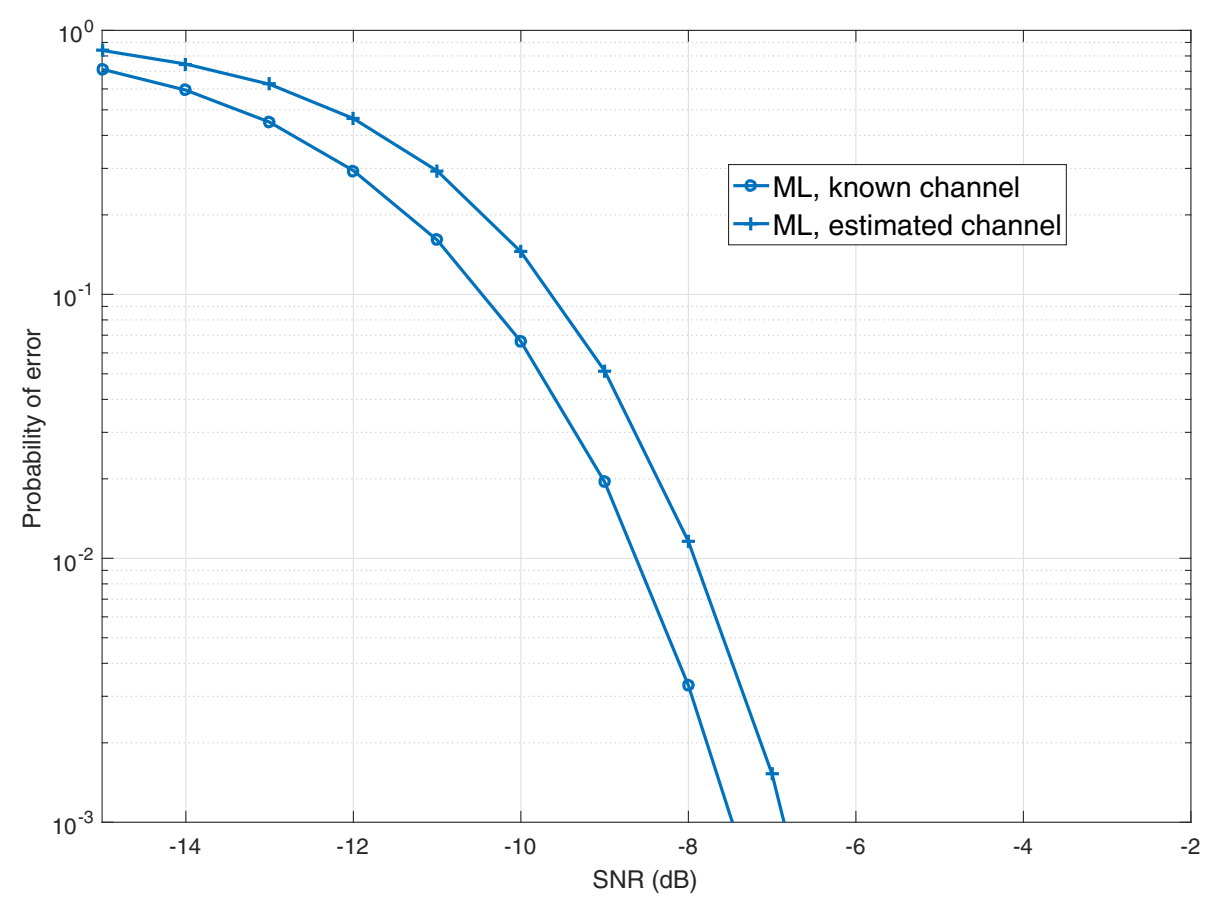

Fig. 5 Error probability versus SNR (dB) of cell ID ML estimator using the suggested DFT-based implementation with channel estimation (20) 


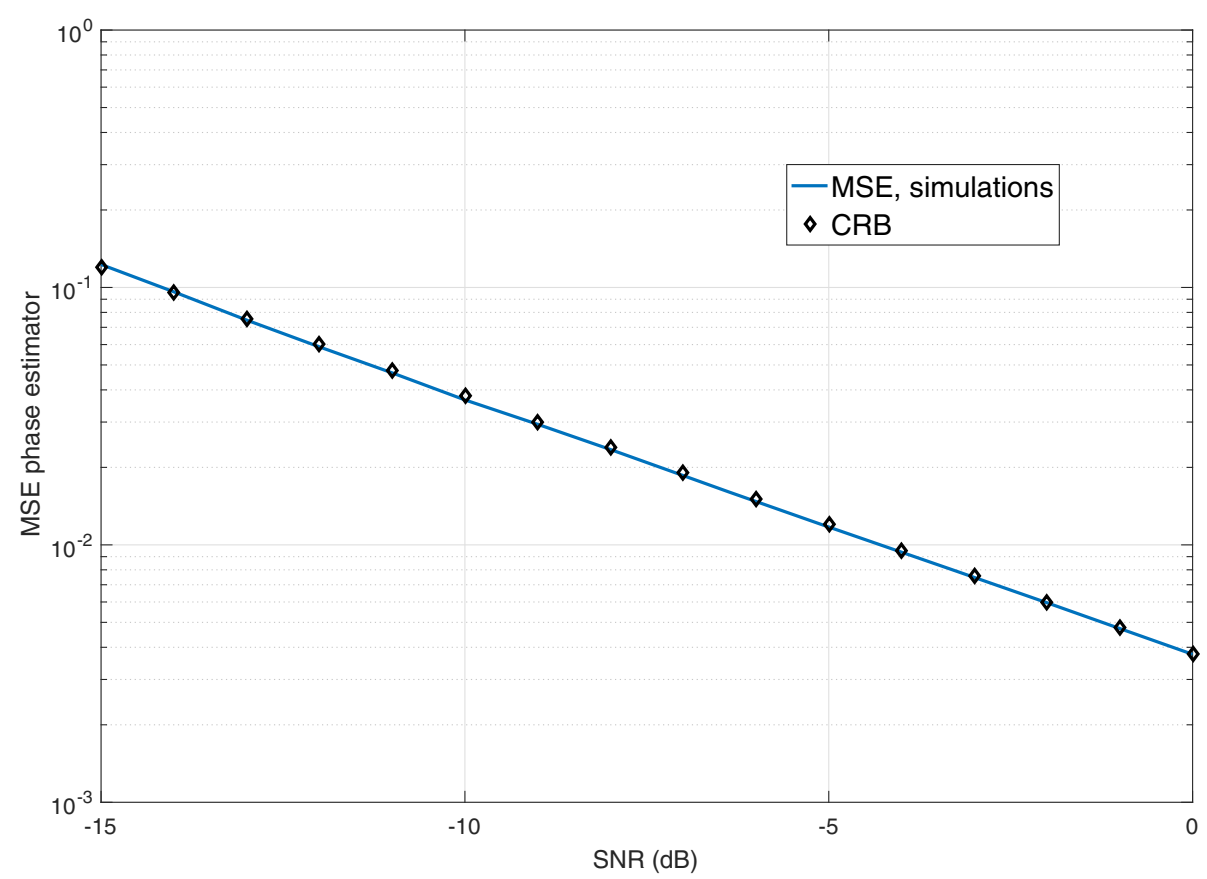

Fig. 6 MSE of channel phase estimation using (18). Comparison with the CRB

estimated, then the alternative ML form (24) can be used without loss of performance compared with (20).

5.2.3 Performance in frequency selective channel

The channel was considered constant over the $180 \mathrm{kHz}$ bandwidth in previous simulations. This is justified by the fact that it can be reasonably assumed that the channel is shorter than the cyclic prefix duration of $4.7 \mu \mathrm{s}$. It results that the coherence bandwidth of the channel is larger than $\frac{1}{4.7 \times 10^{-6}} \approx 212.7 \mathrm{kHz}$, i.e., larger than the $180 \mathrm{kHz}$ bandwidth of the NSSS signal. However, more realistic

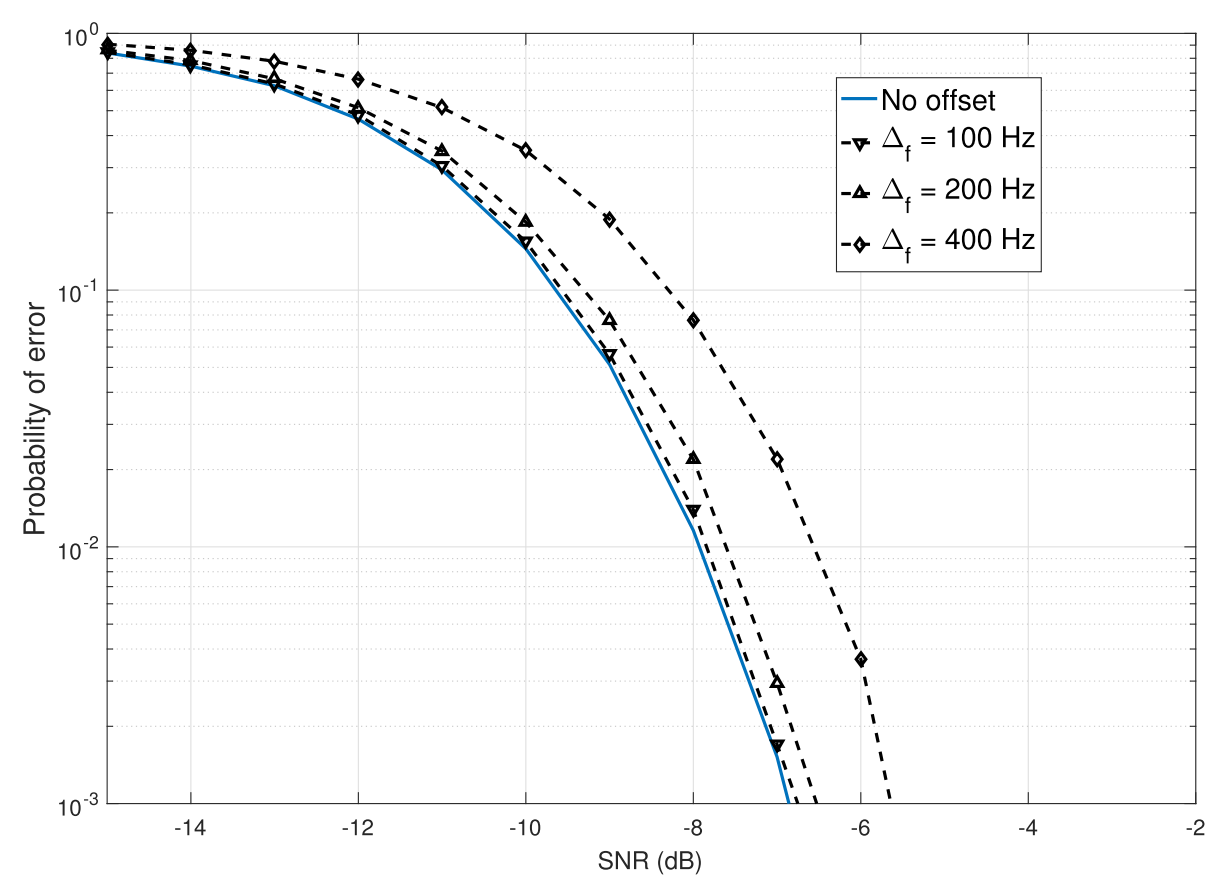

Fig. 7 Error probability versus SNR (dB) of the suggested DFT-based implementation (15) assuming residual frequency offsets $\Delta_{f} \in\{100,200,400\} \mathrm{Hz}$ 


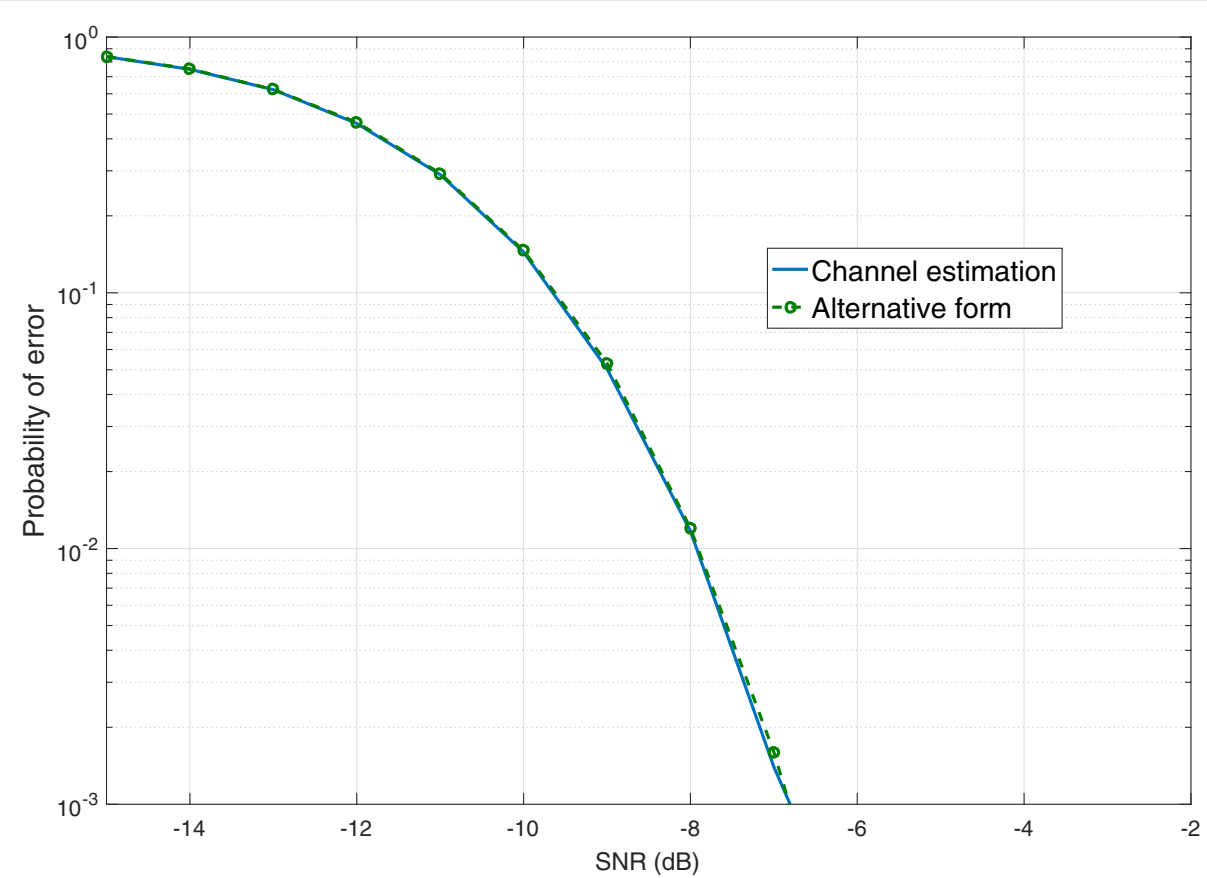

Fig. 8 Error probability versus SNR (dB) of the suggested DFT-based implementation (15) compared with the alternative form

channel models should be considered, taking into account the slight frequency selectivity within the $180 \mathrm{kHz}$. To this end, a four taps channel $\mathbf{h}=\left[h_{0}, h_{1}, h_{2}, h_{3}\right]$ has been simulated, where the coefficients $h_{i}$ are zero-mean complex Gaussian variables (i.e., $\mathbf{h}$ is a Rayleigh channel) with the same variance $\mathbb{E}\left\{\left|h_{i}\right|^{2}\right\}=\frac{1}{4}$. The delay between two taps has been set to $1 \mu \mathrm{s}$, such that the maximum delay is shorter than the cyclic prefix. Note that in that case, (1) should be rewritten to highlight the frequency selectivity. Thus, if the channel is supposed to be temporarily static

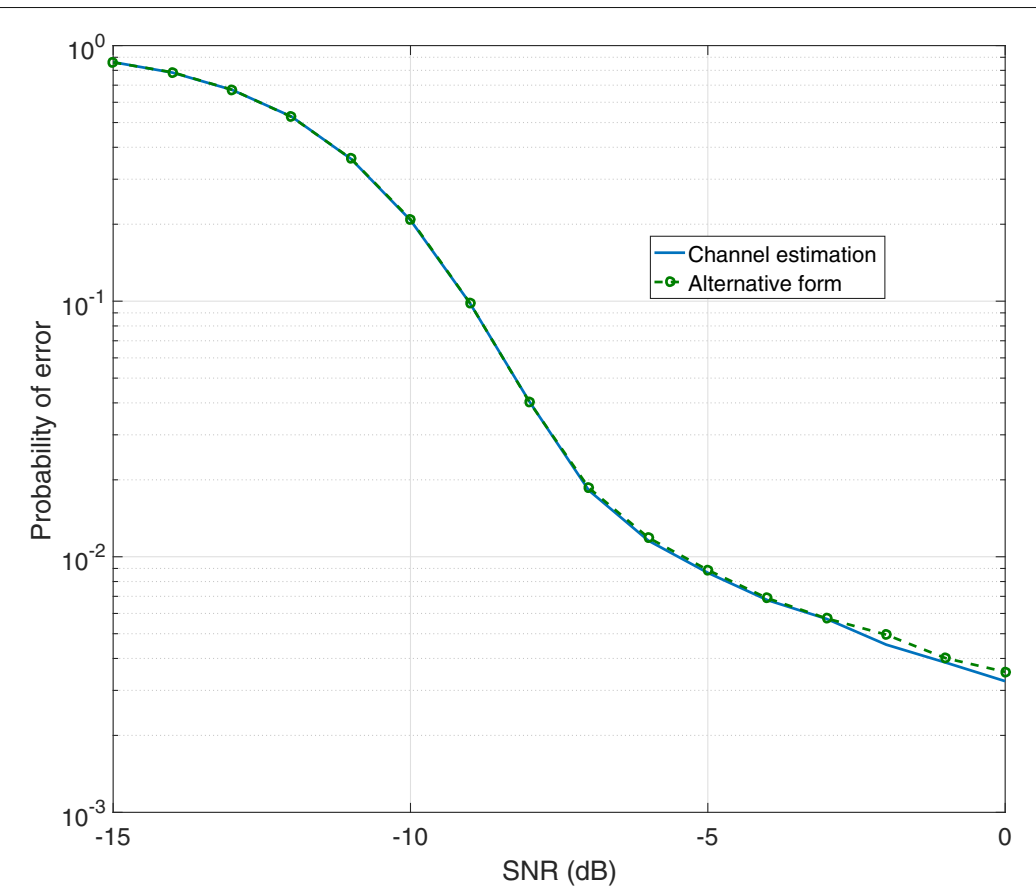

Fig. 9 Error probability versus SNR (dB) of the suggested DFT-based implementation (15) compared with the alternative form in a frequency selective channel 
over the 11 OFDM symbols, then (1) becomes

$$
\mathbf{y}=\overline{\mathbf{H}} \mathbf{d}+\mathbf{w},
$$

where $\overline{\mathbf{H}}$ is a $132 \times 132$ diagonal channel matrix which can be expressed as $\overline{\mathbf{H}}=\mathbf{I} \otimes \mathbf{H}$, with $\mathbf{I}$ the $11 \times 11$ identity matrix, and $\mathbf{H}$ the $12 \times 12$ diagonal matrix containing the channel frequency response $\left[H_{0}, H_{1}, . ., H_{11}\right]$. Therefore, each subcarrier is weighted by a complex coefficient $H_{m}=r_{m} e^{j \phi_{m}}, m=0,1, . ., 11$.

Figure 9 shows the corresponding error probability versus SNR (dB) for both the suggested DFT-based implementation (15) compared with the alternative form. It can be observed that the probability of error is larger in Fig. 9 than in Fig. 8, due to the effect of the frequency selectivity. The error probability is even lower bounded for higher SNR values. Once again, both trajectories match, but this behavior is not further investigated in this paper. However, the advantage of the DFT-based implementation using the channel estimation compared with the modulus form is that it can be adapted to cope with frequency selective channels. Thus, the phase estimation (18) can be redefined for any subcarrier index $m \in\{0,1, . ., 11\}$ as

$$
\hat{\phi}_{m}=\arg \left(\sum_{n=0}^{10} y_{m+12 n} X_{m+12 n}^{*}\left(q, \theta_{f}\right) e_{m+12 n}^{*}(u)\right),
$$

where the sum corresponds to the 11 OFDM symbols. The modulus $r_{m}$ could be also estimated similarly (knowing that ZC sequences have unitary modulus) and integrated in the concentrated-likelihood function to jointly estimate the channel coefficients $H_{m}$ and the cell ID. However, this is not further detailed in this paper.

\subsubsection{Possible use of the method in other applications}

We hereby discuss the possible application of such a suggested method in other applications. As a general comment, it must be noted that the DFT-based algorithm can be used to solve any problem where the root $u$ of a $\mathrm{ZC}$ sequence must be estimated from noisy observations $\mathbf{y}$. For instance in LTE, ZC sequences are used to generate signals in both downlink and uplink. Thus, in downlink, the cell ID is split into two values: one is transmitted through the PSS generated with ZC sequences and the other one is transmitted through the SSS generated with binary sequences [17]. The PSS in LTE is then used for both physical synchronization and part of the cell ID estimation, by estimating the $Z C$ root value $u$ among $\{0,1,2\}$. The suggested technique could then be used to estimate $u$ from PSS, but with some limitations in the presented form. First the reduction of complexity is effective when the set of possible $\mathrm{ZC}$ roots is large, which is not the case as $u \in\{0,1,2\}$. Second, the proposed method requires a prior physical synchronization, and it is not adapted to perform both synchronization and estimation yet.
In uplink-LTE [17] and 5G-new radio (5G-nR) [22], ZC sequences are also used by UEs to generate preambles that are transmitted to start the random access procedure, i.e., to inform the eNBs that UEs intend to access the network. To this end, each UE randomly chooses a preamble among a predefined list, and the corresponding $\mathrm{ZC}$ root $u$ defines a random access preamble identifier (RAPID). This RAPID is then re-transmitted by the eNB to initiate contention resolution among UEs. The eNBs then require to properly estimate the root $u$, and the suggested method could therefore be used to accurately perform this step.

\section{Conclusion}

In this paper, we have presented a DFT-based lowcomplexity estimator of the cell ID in NB-IoT, which is optimal in the ML sense. In fact, the ML estimator leads to a cross-correlation between the observation and a ZC sequence defined as a complex exponential. Thus, by transforming and interchanging the samples of the observation, the cross-correlation can be rewritten as a DFT. It follows that an algorithm for fast computation of DFTs can be used, reducing the complexity of the ML estimator. This principle has been extended to the case where the channel must be estimated. The channel estimate is then introduced in the likelihood function, leading to the maximization of the concentrated likelihood function. This operation can be carried out with a slight additional complexity. The simulation results have shown that the proposed method indeed reaches the performance of the exhaustive ML cell ID search. Furthermore, other series of simulations have revealed that the DFT-based technique is robust to the residual frequency offset, as it experiences $\leq 0.5 \mathrm{~dB}$ loss up to $200 \mathrm{~Hz}$ offset. Further investigations will be undertaken in future works in order to adapt the estimator to frequency selective channels.

\section{Appendix: cramér-Rao bound of $\phi_{\alpha}$ estimator}

The CRB $[20,21]$ is defined as

$$
\mathrm{CRB}=-\mathbb{E}\left\{\frac{\partial^{2} \ln (L)}{\partial \phi_{\alpha}^{2}}\right\}^{-1},
$$

where $L$ is defined in (7), and then

$$
\frac{\partial^{2} \ln (L)}{\partial \phi_{\alpha}^{2}}=-\frac{2}{\sigma^{2}} \operatorname{Re}\left\{\sum_{n=0}^{131} y_{n}^{*}|\alpha| e^{j \phi_{\alpha}} X_{n}\left(q, \theta_{f}\right) e_{n}(u)\right\} .
$$

Since for any $n=0,1, . ., 131$, we have

$$
\mathbb{E}\left\{\operatorname{Re}\left\{w_{n}^{*} X_{n}\left(q, \theta_{f}\right) e_{n}(u)\right\}\right\}=0,
$$

then we obtain $C R B=\frac{\sigma^{2}}{2|\alpha|^{2} N}$ where $N=132$. 


\section{Abbreviations}

AWGN: Additive white Gaussian noise; CRB: Cramér-Rao bound; DFT: Discrete fourier transform; FFT: Fast fourier transform; ID: Identifier; IDFT: Inverse discrete fourier transform; eDRX: Extended discontinuous reception; eNB: Evolved node B; loT: Internet of things; LMMSE: Linear minimum mean square error; LPWA: Low -power wide area; LTE: Long-term evolution; ML: Maximum likelihood; MSE: Mean square error; NB-IOT: Narrowband Internet of things; NPSS: Narrowband primary synchronization signal; NSSS: Narrowband secondary synchronization signal; OFDM: Orthogonal frequency division multiplexing; ZC: Zadoff-Chu

\section{Acknowledgements}

Not applicable

\section{Authors' contributions}

The author carried out both the theoretical developments and the simulations. The author read and approved the final manuscript.

\section{Funding}

Not applicable

\section{Availability of data and materials}

Data sharing is not applicable to this article as no datasets were generated or analyzed during the current study.

\section{Consent for publication}

Not applicable

\section{Competing interests}

The author declares that he has no competing interests.

Received: 8 January 2020 Accepted: 16 March 2020

Published online: 05 April 2020

\section{References}

1. U. Raza, P. Kulkarni, M. Sooriyabandara, Low-power wide area networks: an overview. IEEE Commun. Surv. Tutor. 19(2), 855-873 (2017)

2. K. Mekki, E. Bajic, F. Chaxel, F. Meyer, A comparative study of LPWAN technologies for large-scale loT deployment. Elsevier ICT Express. 5(1), 1-7 (2019)

3. A. Ikpehai, B. Adebisi, K. M. Rabie, K. Anoh, R. E. Ande, M. Hammoudeh, H. Gacanin, U. M. Mbanaso, Low-power wide area network technologies for Internet-of-things: a comparative review. IEEE Internet Things J. 6(2), 2225-2240 (2019)

4. J. Gozalvez, New 3GPP Standard for IoT [Mobile Radio]. IEEE Veh. Technol. Mag. 11(1), 14-20 (2016)

5. R. Ratasuk, N. Mangalvedhe, Y. Zhang, J.-P. Robert, M. Koskinen, in Proc. of CSCN'16. Overview of narrowband IoT in LTE Rel-13, (Berlin, 2016), pp. 1-7

6. J. Schlienz, D. Raddino, Narrowband Internet of Things - Whitepaper, Technical report. (Rohde \& Schwarz, 2016)

7. Y.-P. E. Wang, X. Lin, A. Adhikary, A. Grövlen, Y. Sui, J. Blankenship, Y. Bergman, H. S. Razaghi, A primer on 3GPP narrowband Internet of Things. IEEE Commun. Mag. 55(3), 117-123 (2017)

8. H. Kröll, M. Korb, B. Weber, S. Willi, Q. Huang, in Proc. of WCNCW'17. Maximum-likelihood detection for energy-efficient timing acquisition in NB-IOT (San Francisco, 2017)

9. K. Manolakis, D. M. Gutiérrez-Estévez, V. Jungnickel, W. Xu, C. Drewes, in Proc. of WCNC'09. A closed concept for synchronization and cell search in 3GPP LTE Systems, (Budapest, 2009)

10. Qualcomm, in 3GPPTSG RAN WG1. NB-PSS and NB-SSS design, Technical Report Tech. Rep. R1-161981, (Sophia-Antipolis, 2016)

11. A. Bhamri, L. Zexian, L. Lindh, C. Ribeiro, in Proc. of VTC'2015-Fall. Primary synchronization signal detection method for device-to-device in LTE-Rel 12 and beyond, (Bosto, 2015)

12. Intel Corporation, in 3GPP TSG RAN WG1. Synchronization and cell search in NB-IoT: performance evaluations. Technical Report Tech. Rep. R1-161898, (Sophia-Antipolis, 2016)

13. A. Ali, W. Hamouda, On the cell search and initial synchronization for NB-IoT LTE systems. IEEE Commun. Lett. 21(8), 1843-1846 (2017)
14. Y. B. Li, S. Chen, W. Ye, F. Lin, A joint low-power cell search and frequency tracking scheme in NB-loT systems for green Internet of Things. Sensors. 18(10), 22 (2018)

15. L. R. Rabiner, R. W. Schafer, C. M. Rader, The Chirp z-transform algorithm. IEEE Transactions on Audio and Electroacoustics. AU-17(2), 86-92 (1969)

16. P. Rajmic, Z. Prusa, C. Wiesmeyr, in Proc. of EUSIPCO'14. Computational cost of chirp Z-transform and generalized Goertzel algorithm, (Lisbon, 2014)

17. 3GPP, 3GPP TS 36.211, Physical channels and modulation (Release 14), Technical report. (3GPP, 2017)

18. R. Frank, Polyphase codes with good nonperiodic correlation properties, IEEE Trans. Inf. Theory. 9(1), 43-45 (1963)

19. D. Chu, Polyphase codes with good periodic correlation properties. IEEE Trans. Inf. Theory. 18(4), 531-532 (1972)

20. D. C. Rife, R. R. Boorstyn, Single-tone parameter estimation from discrete-time observations. IEEE Transactions on Information Theory. IT-20(5) (1974)

21. S. M. Kay. Fundamentals of statistical signal processing : estimation theory (Prentice Hall, 2003), pp. 157-214

22. 3GPP, 3GPP TS 38.211, Physical channels and modulation (Release 16). Technical report. (3GPP, 2019)

\section{Publisher's Note}

Springer Nature remains neutral with regard to jurisdictional claims in published maps and institutional affiliations.

\section{Submit your manuscript to a SpringerOpen ${ }^{\circ}$ journal and benefit from:}

- Convenient online submission

- Rigorous peer review

- Open access: articles freely available online

- High visibility within the field

- Retaining the copyright to your article

Submit your next manuscript at $\gg$ springeropen.com 